REVISTA ANDALUZA DE ANTROPOLOGÍA

NÚMERO 19: DÍAS EXTRAÑOS. CÓMO EL COVID-19 TRANSFORMA NUESTRA COTIDIANIDAD DICIEMBRE DE 2020

ISSN 2174-6796

[pp. 67-86]

https://dx.doi.org/10.12795/RAA.2020.19.04

\title{
TRABAJAR EN TIEMPOS DE COVID. FRICCIONES DE LO ESENCIAL EN LA PRODUCCIÓN ALIMENTARIA. EL CASO DE LOS FRUTOS ROJOS EN LA PROVINCIA DE HUELVA
}

Soledad Castillero Quesada

Universidad de Granada

\section{RESUMEN}

La pandemia provocada por el covid-19 está generando un estado de alarma en el que, por primera vez, se consideran bajo la característica de "esenciales" una serie de profesiones y profesionales. Es el caso del sector de la alimentación y las distintas personas y espacios que lo albergan. En Andalucía, coincidió el decreto de confinamiento con el desarrollo de una de las campañas agrícolas que mejor ilustran el funcionamiento de la industria alimentaria hoy, la campaña de los frutos rojos. A través del presente artículo se muestra cómo la declaración de esencialidad de esta campaña por parte de la Administración pública durante el confinamiento de la primera ola, no se tradujo en una conveniente mejora de las condiciones socio-laborales de las personas que trabajan en ella. Siguiendo una metodología cualitativa de corte etnográfico, basada en entrevistas en profundidad a personas trabajadoras del sector, el artículo sirve de análisis en relación a los contrastes surgidos entre la consideración esencial y las circunstancias reales acaecidas durante la actividad laboral.

Palabras clave: Covid-19; Esencialidad; Agroindustria alimentaria; Frutos rojos; Migraciones; Derechos laborales. 


\begin{abstract}
The pandemic caused by Covid-19 is generating a state of emergency in which, for the first time, a series of professions and professionals are considered key workers. This is the case of the food sector and the different people and spaces it encompasses. In Andalusia, the declaration of lockdown coincided with one of the agricultural campaigns that best illustrates the functioning of the agri-food industry today: berry growing season. This article shows how the classification of this work as essential by the Government during the first period of lockdown did not translate into appropriate improvements in the socio-labor conditions of workers in this sector. Following a qualitative ethnographic methodology based on in-depth interviews with agricultural workers, the article analyzes the contrasts that emerged between the classification of this activity as essential and the real circumstances that prevailed during the work carried out.
\end{abstract}

Keywords: Covid 19; Essentiality; Food agribusiness; Berries; Migrations; Labor rights.

\title{
INTRODUCCIÓN ${ }^{1}$
}

La alimentación ocupa un espacio central en el modo de funcionamiento de los pueblos y sociedades. Su producción, distribución y consumo incide de forma directa en el diseño de la economía, el medioambiente, la red de comercios y, en general, en multitud de factores socioculturales que determinan elecciones tales como qué comemos, de qué modo, de dónde procede y esto a su vez estará vinculado a quiénes producen y cómo producen. De ahí que el conocimiento de cómo y por quién ha sido obtenida la alimentación aporta una masa considerable de informaciones sobre el funcionamiento de una sociedad (Contreras y Gracia, 2005). Estas últimas personas, las que producen, han sido consideradas esenciales durante el estado de alarma, y su actividad como primera necesidad, para evitar el cese del abastecimiento alimentario. En las sociedades industrializadas hoy existe un sentir de sobreabundancia alimentaria (Contreras y Gracia, 2005). Bajo este sentimiento y teniendo en cuenta que las hambrunas han ido acompañando los ciclos de la historia, la situación de incertidumbre provocada por el estado de alarma a partir de la expansión del covid-19 generó un temor ante la posibilidad de escasez alimentaria.

1. El presente artículo forma parte de una investigación más amplia enmarcada dentro de una tesis doctoral financiada por el Ministerio de Educación y Formación Profesional, a través de un contrato predoctoral FPU (Formación Profesorado Universitario) iniciado en octubre de 2018 y que durará hasta octubre de 2022. La misma se desarrolla dentro del programa de Estudios Migratorios del Instituto de Migraciones de la Universidad de Granada. 
La alimentación actual se sustenta en un sistema agroalimentario globalizado, crecientemente industrializado y mercantilizado (Castillero, 2019: 114). Es dependiente de mano de obra de distintas latitudes para poder generar excedentes de exportación por lo que el cierre de fronteras y las restricciones de movilidad mantuvieron en vilo a la población durante los primeros días del estado de alarma. Hay generaciones que por primera vez veían en supermercados, hipermercados y tiendas de alimentación estantes vacíos que representaban imágenes insólitas. Estas situaciones conformaron un clima confuso y es que, según Stuart, "los supermercados acumulan productos en stocks para mantener la impresión de abundancia infinita, fundamental para mantener las expectativas en la actual cultura del consumo" (Stuart, 2011: 54). Es ahí donde el gobierno decreta esenciales una serie de trabajos y actividades, como las actividades que se encuentran dentro de la cadena de abastecimiento del mercado y el funcionamiento productivo de bienes y servicios. Aquí se incluyen, por tanto, la producción de bienes y servicios de primera necesidad como son alimentos y bebidas.

En este artículo vamos a analizar las condiciones en las que han trabajado personas encargadas de salvaguardar un cultivo tan simbólico en Andalucía como es el de los frutos rojos. Para ello, vamos a comenzar contextualizando la producción y comercialización alimentaria hoy para ir describiendo las características de la industria de los frutos rojos y poner en diálogo los discursos de las personas, consideradas esenciales, que han formado parte de la investigación.

\section{METODOLOGÍA Y OBJETIVOS}

El siguiente trabajo se basa en la investigación de campo realizada durante la campaña de recogida 2019-2020 y durante la temporada de plantación de la fresa para la campaña 2020-2021. Como fuentes principales se exponen las entrevistas en profundidad llevadas a cabo a trabajadoras y trabajadores del sector, tanto recolectoras como agricultores en calidad de propietarios de las explotaciones. Los objetivos del trabajo son, por un lado, contextualizar los modos de funcionamiento de la producción alimentaria hoy a partir de la ilustración del sector de los frutos rojos y, por otro lado, mostrar cómo el reconocimiento de esencialidad no garantiza medidas sociales básicas. A partir de una metodología cualitativa de corte etnográfico, se triangulan extractos de una serie de entrevistas en profundidad que darán cuenta del modo en que se realiza el trabajo en este sector.

\section{INSERCIÓN DE LA ALIMENTACIÓN EN LA INDUSTRIA ALIMENTARIA GLOBAL}

El estudio de la alimentación ha de rebasar el hecho biológico, pues la elección humana sobre los recursos alimenticios no es solo de carácter fisiológico, sino cultural. No comemos lo que comemos solamente para saciar nuestra hambre o para adquirir el 
número indicado de calorías diarias para un correcto funcionamiento de nuestro organismo (Castillero, 2019: 114). Como expone Medina:

"La alimentación es necesaria para la supervivencia, pero, más allá de eso, es particular y significativamente utilizada por los individuos y los grupos como una vía de identidad privilegiada, como una estrategia social para estar juntos, para establecer y celebrar lazos, para apaciguar la nostalgia y para convertirse en un escaparate frente a los 'Otros' o en una estrategia económica de supervivencia' (Medina, 2014: 33).

Para hablar sobre producción alimentaria hoy, necesitamos situarnos dentro de la industria alimentaria global. Esto es, dentro de los procesos que tienen lugar desde la siembra en la tierra, la transformación, el transporte, la comercialización y su colocación en los espacios de consumo y las características de los mismos, todo lo cual se encuentra inserto en un proceso de globalización. Es difícil situar el comienzo de la expansión de los mercados más allá de sus fronteras, pero podríamos marcar el inicio en paralelo al surgimiento de la idea de globalización. Aunque podría considerarse un concepto relativamente nuevo, hay quienes, como Walter Mignolo (1998:32), relacionan el concepto de globalización y la expansión occidental iniciada desde 1500 (Contreras y Gracia, 2005: 404). Podríamos situar aquí una referencia a ese intercambio y domesticación de plantas, así como la introducción de nuevas especies a un lado y al otro del Atlántico. Productos como el maíz, el chocolate, la vainilla, el tomate, el pimiento o el aguacate fueron productos introducidos desde distintas zonas de América a Europa (Albán, 2010: 16). En ese sentido, la tradición musulmana presente en Andalucía poseía las condiciones necesarias, hábitos culturales e infraestructuras para que fuera en sus huertas donde se ensayasen y aclimatasen nuevos productos. Esto explica la rápida difusión de productos como el pimiento (Moreno Gómez y Amado, 1998: 264).

A partir de esta inserción global, la alimentación pasa a formar parte del mercado que decide la inclusión/exclusión de territorios y, por tanto, de personas (Moreno Navarro y Delgado, 2013: 62). Aunque el valor de los alimentos como transacción, intercambio y mercancía no es exclusivo de las sociedades contemporáneas, en la actualidad los intereses privados que se ejercen sobre el suelo y las materias primas empeoran el acceso a la tierra y la redistribución alimentaria equitativa (Gracia, 2015). Esto explica cómo, a pesar de que nunca las sociedades occidentales habían tenido tanta alimentación disponible como en la actualidad, sin embargo, esta alimentación no es accesible a toda la población existente. La globalización alimentaria ha ido transformando la manera en cómo producimos y nos alimentamos, centralizando una producción que genere excedentes para la exportación y no una producción cuya prioridad sea el abastecimiento de la población. Por eso, una de las características esenciales de la industria alimentaria global es la modernización de la agricultura para una superproducción que sea capaz 
de comercializar a escala planetaria (Entrena, 2008). A continuación, vamos a concretar estas características en el contexto andaluz.

\subsection{Producción alimentaria en Andalucía hoy. Algunas cuestiones clave}

Andalucía es una región situada al sur del Estado español que se encuentra a tan solo 13 kilómetros del continente africano. Esto ha hecho que, por su condición mediterránea y atlántica, siempre haya sido una puerta al mundo, un lugar de paso, personas y mercancías.

El sistema productivo andaluz actual se corresponde con el modelo propio de la agroindustria alimentaria global: atiende a la regla de menor coste para un mayor beneficio (Castillero, 2019: 114). Se trata de una agricultura intensiva enfocada a altos niveles de exportación para ser competitiva en los mercados. Para ello, en muchas regiones se ha optado prácticamente por el monocultivo, como es el caso de la provincia de Huelva en la que hasta hace unos años el principal y casi exclusivo producto era la fresa, diversificada por otras variedades como el arándano o la frambuesa, en los últimos años. La climatología de zonas como Huelva o Almería, y la cantidad de horas de sol de las que disponen al año, han permitido jugar con técnicas de cultivo tales como la agricultura bajo plástico o invernadero. Según el informe de la Balanza Comercial Agroalimentaria de Andalucía correspondiente al mes de abril (2020), en el periodo enero-abril se han incrementado las exportaciones con respecto a 2019 en un $1,7 \%$ en valor y un $0,3 \%$ en peso, consiguiéndose, según el informe, el mejor dato en peso de productos agroalimentarios exportados y el segundo en valor desde que se tiene registro de datos. Traducido en cifras hablaríamos de 3,2 millones de toneladas y 4.559 millones de euros los exportados en productos agroalimentarios. Esto da cuenta de que la producción alimentaria no ha cesado durante el estado de alarma ni el confinamiento de la primavera del 2020, sino que además se ha incrementado la producción alcanzando récords históricos.

Por el contrario, Andalucía no cuenta con una red de industria de manipulación, transformación y envasado sólida, sino que es el territorio en el que se produce la materia prima y es exportada para continuar con el resto de procesos. Esto le hace, en palabras de Manuel Delgado, “jugar un papel subordinado y dependiente con los centros industrializados siendo su función abastecer de mano de obra y la explotación de los recursos naturales, a disposición del crecimiento" (Delgado, 2013: 103). Un crecimiento cuyos parámetros han sido en gran parte los económicos y donde se dificulta la estabilidad laboral, pues no hay unas condiciones fijas respecto a las cuales las personas puedan organizar su vida. La alta variación respecto a los días trabajados, así como la flexibilidad en los horarios, provocan que sea la mano de obra la que deba adecuarse a las necesidades en cada momento (Delgado, 2013: 75). Esto ha ido creando, por un lado, desempleo en la población local que opta incorporarse a otros sectores dentro o fuera del territorio y, por otro lado, la incorporación de trabajadoras y trabajadores migrantes dispuestos a aceptar unas condiciones más laxas debido a su vulnerabilidad. 
Descritas algunas características y dimensiones de la industria alimentaria global a escala general, y haciendo mención de las mismas en Andalucía, vamos a exponer algunas de las situaciones experimentadas por personas consideradas esenciales durante el estado de alarma para garantizar que el modelo productivo no cese. Para ello, analizaremos el término "esencial" en sí, en relación a la situación presente.

\section{RELATOS ESENCIALES Y SUS FRICCIONES. LA TEMPORADA DE LOS FRUTOS ROJO COMO EJEMPLO}

La pandemia provocada por el covid-19 ha transformado el análisis de distintos escenarios y agentes, así como los modos de nombrarlos. Es el caso de quienes trabajan en los primeros eslabones de la cadena de producción alimentaria, que por primera vez han pasado a denominarse como personas esenciales. El sector agrícola se denomina así elemental en un estado de alarma donde una de las preocupaciones de los gobiernos de distintos países ha sido garantizar la actividad agroalimentaria para proteger la cadena de suministro.

\subsection{Sobre el concepto de esencialidad}

Según el diccionario de la RAE (2020), "esencial" significa perteneciente o relativo a la esencia. A su vez, "esencia" se define como aquello que constituye la naturaleza de las cosas, lo permanente e invariable de ellas o lo más importante y característico de una cosa. Sin duda, los seres humanos por más diversos que sean entre ellos, en su naturaleza primaria comparten el hecho de alimentarse. Por ello, el sustento alimentario es la esencia de la vida, es lo más importante para que esta sea posible. Sin embargo, en términos jurídicos y sociales, un servicio esencial es un concepto indeterminado que implica una valoración en cada etapa histórica, en relación a los intereses económicos y sociales de la comunidad (Fernández García, 2006:329). En este momento histórico lo esencial ha sido, entre otras cosas, garantizar la continuación de la producción alimentaria. A su vez, esta centralidad ha visibilizado la urgencia de pensar la alimentación como un derecho a la soberanía alimentaria, amparado por un sistema productivo orientado a las necesidades reales de la población y no al mercado (Moreno Navarro, 2013: 58). Es decir, interpretar la alimentación como esencial y no como una hoja de balance donde todas las demás consideraciones desaparecen (Stuart, 2011: 45). Cuando hablamos de todas las demás condiciones hablamos de una producción en condiciones de trabajo dignas, donde las trabajadoras y trabajadores tengan sus necesidades cubiertas, pues son esenciales para cubrir las nuestras. Sin embargo, y a pesar de que existe lo que se conoce como principio de precaución-presente en el artículo 191 del Tratado de Funcionamiento de la Unión Europea (Eur.Lex., 2020), el cual exige que en caso de amenaza para el medio ambiente o la salud y en una situación de incertidumbre científica se tomen las medidas apropiadas para prevenir el daño-, se ha permitido que trabajadores y trabajadoras esenciales no cuenten con mascarillas, equipos de protección ni salubridad necesaria. De ahí que 
hablemos de fricciones, pues no se han tenido en cuenta unas características esenciales para las propias personas esenciales, o lo que es lo mismo, la esencialidad no fue extensiva a sus condiciones vitales.

En Andalucía, el estado de alarma coincidió con la campaña del sector de los frutos rojos, uno de los más significativos en su agricultura, tanto en extensión como en cifras de producción y exportación. De ahí que resulten más significativas las fricciones en un sector que presenta los datos que se muestran a continuación.

\subsection{Valor numérico del fruto rojo en Andalucía}

Cuando hablamos de fruto rojo hablamos de la producción de fresa, arándano, mora y frambuesa. La temporalidad de cada cultivo varía y, en este caso, es la recogida de la fresa junto con el arándano la que se ha visto en el epicentro de la pandemia. Se trata de un sector muy influyente en Andalucía, pues representa la primera región exportadora de fresas en el mundo. Su extensión ocupa una superficie de 11.700 hectáreas, según datos de la Asociación Onubense de Productores y Exportadores de Fresas de Huelva (FRESHUELVA, 2020), en la campaña de 2019-2020, aumentando un 2\% respecto a la campaña anterior, donde el sector ocupaba unas 11.464 hectáreas. De entre las distintas frutas, la fresa sigue siendo la producción más consolidada, ocupando 6.843 hectáreas en la campaña de 2019 y produciendo unas 303.215 toneladas, según datos del Observatorio de Precios y Mercados de la Junta de Andalucía (2019). La fresa constituyó en la campaña de 2018, según el citado informe, el 3,9\% del valor de la Producción Vegetal y el 3,2\% de la Producción de la Rama Agraria Andaluzas. Detrás de la fresa se situaría la producción de arándano, un cultivo que aumentó en un $29 \%$ con un total de 2.842 hectáreas en 2019, alcanzando una producción de 51.569 toneladas. Esto sitúa a Andalucía como la primera región productora y exportadora de arándano de España y de la UE. Por tanto, es común que cada vez más los agricultores diversifiquen las hectáreas de producción y, además de fresa, cultiven arándano y frambuesa. Aunque esta última no ha alcanzado aún los niveles de las anteriores, se trata de un cultivo en expansión igualmente. España es el segundo productor de frambuesa en la UE con una cuota de participación en la producción del 20,3\%, situándose casi por completo en la provincia de Huelva, donde se estima que la superficie en seis años ha aumentado en un $180 \%$. En la campaña de 2018, según datos del Observatorio, la superficie de frambuesa ocupaba 2.433 hectáreas, produciendo unas 43.794 toneladas. El grueso de las exportaciones andaluzas de fresa va destinado a la Unión Europea, siendo los principales mercados Alemania, Reino unido, Francia e Italia. En el caso del arándano, igualmente el 99\% de las exportaciones van destinadas a la UE: Alemania, Reino unido y Países Bajos son los principales consumidores. En cuanto a la frambuesa, de igual modo las exportaciones se destinan a países europeos, siendo Reino Unido el primer mercado, seguido de Alemania con un 26,4\%. 
Es muy importante no perder de vista estos datos porque el volumen de crecimiento de las hectáreas cultivadas y, por ende, el volumen de las exportaciones, explican cambios significativos tanto en las transformaciones del territorio, en la economía de los pueblos, así como en los flujos de trabajadores y trabajadoras presentes.

\subsection{Transformación del territorio y el perfil de los trabajadores y trabajadoras}

La experiencia del sector de los frutos rojos desborda la clásica sectorialización de la actividad económica. La mayor parte de la fresa producida en Huelva y consumida en la Unión Europea tiene su origen en la reproducción de sus semillas en los campos de experimentación y laboratorios de las universidades del Condado de Monterrey, California (Márquez Domínguez, 2016: 606). A su vez, estas plantas ya libres de enfermedades son adquiridas por empresas y cooperativas europeas que las multiplican en viveros principalmente de la provincia de Ávila donde, una vez fortalecido el estolón de la planta, serán sembradas, en este caso en la provincia de Huelva (Márquez Domínguez, 2016:606). Por ello, hablar de la producción de frutos rojos en la provincia de Huelva es a su vez hablar de una producción multisituada, en la que entran en juego distintos agentes y territorios. La intensificación de la producción con fines exportadores ha implantado nuevas políticas de contratación, especialización y fragmentación de los mercados de trabajo, gestión de una mano de obra deslocalizada, así como una creciente feminización para unas tareas determinadas. La convergencia de estas características está presente en las cadenas agrícolas globales (Reigada, 2012). Los inicios de la expansión del sector fueron en paralelo al crecimiento de otros sectores en Andalucía como la construcción o el sector servicios, lo que ha provocado un cambio en el perfil de trabajadores y trabajadoras. Así, Gualda Caballero (2003) habla del paso del jornalero andaluz al campesino migrante en este tránsito. No obstante, no hay un corte sectorial entre nacionalidades o perfiles de trabajadores y trabajadoras, sino que han ido y siguen conviviendo, pues las crisis cíclicas provocan idas y venidas constantes al campo cuando caen otros sectores. Así lo exponen varios agricultores con los que hemos ido trabajando durante el trabajo de campo, como Enrique $^{2}$, un joven agricultor de Villablanca que desde hace 9 años gestiona una finca con fresa, frambuesa, arándano y algunos aguacates:

"En el estado de alarma vino muchísima gente de bares, gente de..., de todo, gente que tú las ves en el pueblo y mujeres que tú las ves digamos "pimpolleás", muy arregladas y ahora han tenido que venir al campo porque tienen que comer y yo nunca les he puesto falta. Esto es de mi abuelo, una tierra que cogimos en herencia y cuando cayó la construcción hace nueve años, mi padre cogió una depresión y yo dejé los estudios y dije: venga papá, aquí hay que hacer algo y empezamos y nada, pero vaya, esto lo llevo todo yo" (Diario de campo, octubre 2020).

2. Enrique es el pseudónimo que se utiliza para preservar la intimidad del participante. 
Hay una línea directa entre la caída de una burbuja y la incorporación a otra, en este caso a los frutos rojos. Pasó con la caída de la construcción y, como relata Enrique, ha vuelto a pasar con el estado de alarma. Al respecto de esa regresión al campo desde la construcción, Juan ${ }^{3}$, un agricultor de Lepe, cuenta:

"Mira, la verdad que yo me acuerdo los últimos años que estuve en la cooperativa: había gente que, [desde] la albañilería, se metieron en el campo, a lo mejor ponían un poquito de fresa, un poquito de frambuesa, bueno, pues eso ha desaparecido ya porque el que es de campo es de campo y quien no, no. La gente vendiendo arcos, invernaderos, vendiendo todo porque no puedes hacer igual que en la albañilería el viernes a mediodía hasta el lunes. Esto en el campo no existe y el que lo haga va de culo. Si eres de cítricos a lo mejor, pero los frutos rojos no. Y lo que es fresa y frambuesa eso es todos los días tiene algo: si no tiene botritis tiene cenizo, sino arañas, todos los días tiene algo" (Diario de campo, octubre 2020).

Juan alude a la constancia del campo y la dureza del mismo como condiciones que hacen que no todo el mundo pueda participar y subsistir de este sector. La falta de mano de obra es una constante a la que remiten las distintas personas entrevistadas, como Adolfo, dueño de una explotación que cuenta a su vez con un almacén de manipulado:

"La problemática de Huelva es la mano de obra, haya covid o no haya covid. Mano de obra local hay poca, no quieren trabajar. Está por el sector servicios, la construcción, los subsidios por desempleo. Los trabajadores que vienen de fuera repiten al $90 \%$; ya conocen a la manijera y se ponen en contacto con la manijera" (Diario de Campo, octubre 2020).

Sin embargo, esa idea de "no quieren trabajar", que explicita Adolfo, es interpretada de un modo distinto por algunas de las trabajadoras participantes en la investigación. Por ejemplo, Alicia, trabajadora en este caso del manipulado que se lleva a cabo en los almacenes donde llega la fruta del campo y se selecciona, envasa y etiqueta para el mercado, apunta:

"Las horas son impredecibles, depende mucho de la empresa, yo he pasado por muchas empresas y lo que está claro es que se sabe cuando entras, pero no cuando sales. Es que es todo $m u$ variable. Hay empresas que se han modernizado mucho y han puesto turnos, pero volvemos a lo mismo, lo mismo un día trabajas quince horas y al otro no trabajas o volvemos a lo mismo. Cuando la producción está en su máximo ponen turno de mañana y de tarde y tú te puedes organizar: cuando estás

3. Juan es igualmente un pseudónimo. Se especificará siempre que se utilice un pseudónimo, no siendo necesario cuando se muestre el nombre real del participante o la participante. Los lugares donde se ubican las fincas y las personas entrevistadas siempre van a corresponder con la realidad para que ella persona lectora pueda tener una dimensión y ubicar distintos territorios donde se asienta esta producción. 
en el turno de mañana, pero no de tarde que estás hasta que acabas y esas horas se pagan normales. Aquí horas extras, domingos, festivos y plus por nocturnidad, nada" (Entrevista 1) .

Juan, como agricultor, entiende que es natural no descansar pues la naturaleza no descansa y Alicia, como trabajadora, requiere de una organización vital en base a su trabajo por lo que para ella esa idea de impredecible se le torna como un aspecto negativo. La visión de Alicia coincide con la de Ana, quien ha sido trabajadora del fruto rojo durante 16 años:

"Te obligan a trabajar de lunes a domingo y a echar hasta las cinco o las seis de la tarde y habría que repartirlo y organizarlo. Si a ti te toca este domingo, descansas el sábado y te puedes organizar y ¿qué pasa? Pues que la gente de aquí no tenemos la misma necesidad que la que viene de fuera. Mira, la gente de fuera y la gente de aquí te dicen que vas a echar horas y tú te alegras porque para la mierda que ganas. Pero la gente de aquí son las menos y esa es la realidad. La gente que verdaderamente tiene una necesidad lo pasa muy mal a la hora del trabajo, asustada no sea que la echen" (Entrevista 2)

Citando a Isidoro Moreno, podríamos interpretar las palabras de Ana cuando habla de gente "de aquí" y "gente de fuera" como el mapa dibujado por la exclusión social donde "dentro de cada país, estado y ciudad se "territorializa" y "sectorializa la riqueza, el bienestar, la pobreza y la marginación” (Moreno Navarro, 2013: 65). Las exigencias del sector han crecido de forma exponencial al crecimiento de las hectáreas, la producción, las demandas y, por tanto, la expansión del mercado agroalimentario. A mayor producción, mayor exportación, mayor necesidad de mano de obra y, en consecuencia, mayor dedicación traducida en número de horas y días de la semana trabajados.

A finales de la década de los 90 comienza a implantarse lo que se ha conocido como los contratos en origen, cuya primera cláusula es el retorno de las personas participantes una vez hayan terminado el periodo de trabajo. Ante un aumento de la superficie cultivada y una mano de obra que se desplaza a otros sectores, los agricultores viven la inseguridad de poder abarcar la temporada. Es desde el Ayuntamiento de Cartaya que se inicia la idea de traer población, principalmente mujeres, primero de países del Este y más tarde de Marruecos. La entrada de países como Rumanía o Bulgaria en la Unión Europea hizo que no fuesen necesarios este tipo de contrataciones, pues sus ciudadanos pasaban a poder tener movilidad por el territorio. En un principio, la contratación en origen forma parte

4. Entrevista 1: realizada a Alicia, pseudónimo utilizado para preservar el anonimato de la participante en Mazagón. 26 de octubre de 2020, 17:00 p.m., 45:16m. Grabadora de voz.

5. Entrevista 2: realizada a Ana en Escacena del Campo. 28 de octubre de 2020, 12:20 p.m., 50:06m. Grabadora de voz. 
del programa AENEAS (Programa de Asistencia Técnica y Financiera a Terceros Países en Emigración y Asilo). Dicho programa nace en 2004 con financiación de la Unión Europea, con el objetivo de luchar contra lo que consideran inmigración clandestina y controlar así los flujos migratorios (Arab, 2020:77). Al programa AENEAS le siguió el proyecto M@RES (Sistema para la movilidad de flujos migratorios de trabajadores en la provincia de Huelva) (Arab, 2020: 74). Este modelo de contratación ha sido, por un lado, considerado como un modelo ideal y exportado a otros territorios. Por otro lado, ha recibido numerosas críticas, pues se basa en la contratación de mujeres vulnerables, pues uno de los principales requisitos es que tengan hijos/as o cargas familiares que aseguren la vuelta tras el final de la campaña. Esto limita la aceptación de su presencia y circulación en el territorio exclusivamente a su condición de temporeras. A partir de 2001 es cuando se entiende como consolidado este sistema de contratación, con 7.000 trabajadoras para la campaña de 2001-2002, que ascendieron a unas 35.000 contrataciones en la campaña de 2005-2006 con la contratación de mujeres principalmente de Polonia y Rumanía (Reigada, 2011). Y es en la campaña de 2006-2007 cuando se dan cambios significativos con la contratación de mujeres en origen de Marruecos, contrataciones que siguen realizándose hasta hoy.

A la mano de obra de las contrataciones en origen, se le suma la presencia de las mujeres y hombres de Europa del Este, personas de distintas partes del continente africano, que a la vez son trabajadoras en diversos cultivos tanto a nivel andaluz como nacional, así como trabajadoras y trabajadores autóctonos. Encontramos una población plural encargada de la recolección, manipulado y mantenimiento del sector.

A continuación, nos centramos en las vivencias y relatos de distintas personas que han estado presentes en una de las temporadas más atípicas que se recuerdan, durante el estado de alarma.

\subsection{El trabajo del fruto rojo y el covid-19}

La temporada de fresa comienza a finales de septiembre con la plantación en los lomos, que son las construcciones de tierra donde amarra la planta y se suele extender durante el mes de octubre. Las variedades más tempranas pueden comenzar a dar fruta en diciembre, pero lo común es que la fresa comience a producir desde finales de enero a junio, siendo los meses más importantes los de enero, febrero y marzo, pues es cuando más valor tiene la fruta en el mercado porque está recién llegada. Estos meses coinciden con la producción de arándano, que, jugando con las variedades plantadas, tempranas y tardías, se van sucediendo hasta llegar incluso hasta julio. Ya en mayo los mercados tienen la cantidad de fruta como para abaratar los precios y deja de ser tan rentable como producto, lo cual no quiere decir que no se siga extendiendo la campaña. Como se observa, los meses imprescindibles para su recogida coinciden justo con los meses del estado de alarma y el confinamiento. Por tanto, la campaña supuso un revulsivo social 
tanto para empresarios y agricultores (normalmente hombres), como para trabajadoras y trabajadores. Aun así, tanto los datos estadísticos como los discursos de los agricultores muestran cómo, a pesar de las inclemencias del tiempo, se ha conseguido salvar la temporada. Datos de FRESHUELVA indican que se han exportado en la campaña de fresas de 2020 un total de 250.000 toneladas, lo cual supone un retroceso del $20 \%$ respecto al año anterior, derivado de la situación vivida por el covid-19 y la falta de mano de obra. Sin embargo, la misma institución afirma que ha sido el arándano el producto que ha mantenido la base de la producción para los agricultores que hayan diversificado sus productos. No obstante, los agricultores entrevistados exponen no haber tenido una bajada en la producción. Incluso hay quienes afirman que ha sido un año atípico porque ha faltado fruta y el precio ha caído. Es lo que sostiene Juan, agricultor de Lepe:

"Este año ha faltado fruta y ha valido más. Menos mano de obra, ganas más dinero, es que se ha alargado más porque se ha cogido hasta el último. Este año ha sido lo contrario, lo que no ha prevenido nadie. Este año por el tema del clima ha habido menos producción y el arándano vale más dinero, lo que no se esperaba nadie. Un tiempo de neblina o lo que sea en la floración y no ha cuajado y este año ha faltado fruta. Es mejor coger menos y que valga más. Este año se ha alargado más porque se ha cogido hasta el último. Con la planta tardía he cogido yo hasta el 17 de junio. Si lo normal es vender a tres euros o cuatro los últimos, ahora se han cobrado a seis y medio porque no había”. Juan señala un factor que es clave en la producción alimentaria, exista o no el estado provocado por el covid-19: la influencia y determinación de la climatología en la planta y en el fruto. Según Moreno Gómez y Amada:

"En términos históricos, los dos factores que influyen decisivamente en el consumo de los diferentes tipos de alimentos son la clase social y las condiciones climáticas que determinan la producción, y esto último es tanto más así por la dificultad del transporte y la falta del excedente necesario" (Moreno Gómez y Amada, 1998: 264).

La situación descrita pdeja ver cómo la sobreproducción influye en la caída de precios $\mathrm{y}$, por tanto, una producción más sostenible, donde se produzca lo necesario, dará valor a los precios y a las condiciones de trabajo. Aunque apunta a que revierte en menos mano de obra el hecho de producir menos, lo cierto es que, como muestran los relatos de agricultores entrevistados, en la campaña se ha trabajado con el mismo número de personas, pero durante más horas. Así, a mi pregunta dirigida a Adolfo sobre cómo han logrado salvar la temporada, su respuesta es tajante: "echando más horas" (Diario de campo, octubre 2020). El propio Juan afirma que él tiene siempre gente trabajando porque

"La verdad que yo tengo la gente de otros años y eso porque yo echo un montón de horas y la gente viene a ganar dinero, lo que las inspecciones de trabajo no quieren 
porque ya tienes que descansar un día a la semana, no pueden venir por la tarde y la gente lo que quiere es ganar dinero" (Diario de campo, octubre 2020).

Volvemos a encontrar contradicciones con el discurso de Alicia y Ana que, como trabajadoras, ven en la ausencia de días libres, descanso y planificación el problema de la escasez de mano de obra que otros, como Adolfo, señalan.

A la pregunta de si han tenido bajas sustanciales, Jaime, un agricultor de Lepe que, junto a su padre, suelen tener en campaña a unas 25 personas trabajando, afirma que:

"Nosotros la verdad que bajas no tuvimos muchas, pero sí un par de ellas y hay algunos que se mueven en el pueblo y no dejan de venir, la gente no para, aunque tenga el covid. Los morenos ${ }^{6}$ dicen no, nosotros trabajar, eso no pasa nada. Digo, desde luego. Hasta ahora hemos estado $m u$ tranquilos en Lepe. Es verdad que cada vez hay más contagios. Antes la cuadrilla que venía de fuera le tuve que poner autobuses: date cuenta que si están acostumbraos en venir en tres coches, ahí tenían que venir en seis. Dos furgonetas que teníamos de esas grandes daban dos vueltas. Fue en plena producción, fue el peor momento, venía la gente de Sevilla" (Diario de campo, octubre 2020).

Al igual que Juan, el relato de Jaime deja ver cómo ha habido una incorporación de nuevos actores que se han vuelto esenciales y para los que a la vez el campo se les ha presentado como una oportunidad esencial ante la caída de sus nichos de empleo anteriores. Además de gente de otros sectores, la base ha sido la población que repite cada año y que es asidua al sector. Alicia, como trabajadora del manipulado, cuenta como el covid y la situación le hicieron abandonar su puesto de trabajo:

"Bueno, durante el estado de alarma, cuando llegó el covid, yo soy una persona de riesgo y yo salí huyendo de allí porque yo vi que no había ningún control ni medida de seguridad. Después, en teoría, pusieron un protocolo aunque yo el protocolo sinceramente..., a mí mis compañeras me mandaban audios, me mandaban fotos, me mandaban vídeos y yo ahí no veía..., y después que también ha habido casos de covid y tapados de personas que yo conocía y ¿has avisado a la empresa? Sí, lo saben y ¿ por qué no han avisado a las compañeras? No sé, estamos hablando de un peligro de salud a nivel mundial, o sea hay que tener cuidado con estas cosas, hay que informar, pero ¿qué pasa? Les damos igual. Realmente somos números; realmente, para ellos, lo único que interesa es ganar dinero, la producción. Y entonces ahí me fui y ahí lo perdí ya todo. Con el covid, siendo una persona de riesgo y viendo que ni en los campos ni medidas de seguridad, ni distancia, no tenían agua para lavarse las manos y en las cooperativas no se hacían controles de temperatura ni te daban

6. "Moreno" es el término con el que designan comúnmente a las personas de distintos países africanos. No se entiende como forma despectiva pues la propia población africana se autodenomina "morena". 
guantes ni mascarilla, que no había una cosa muy controlada, entonces yo no me quería poner en la boca del lobo" (Diario de campo, octubre 2020).

Aún con la situación que relata Alicia, el sector no ha cesado ni un solo día, sino que, como hemos visto, ha recibido una mayor afluencia de gente en un contexto de más peligrosidad. Por cuestiones económicas, la población queda desprotegida. En este caso se sufren inclemencias sanitarias que afectan a sectores que se han instaurado para satisfacer necesidades de mercados foráneos sin garantizar las coberturas de unas condiciones y sistemas de trabajo reforzados para la población autóctona. El decretar como esenciales ciertas actividades y a las personas que se dedicaban a ello, tenía como objetivo no solo el que no cesara la actividad si no que, en todo lo posible, se protegiesen también las maneras de producir. Y al parecer ha sido posible, en palabras de Enrique:

"En abril o marzo o por ahí empieza a producir Marruecos y baja el precio porque los mercados de fuera compran a Marruecos y este año cortó, en esta campaña que corte de nuevo. Ha ido el año malamente porque hemos tenido que trabajar con mascarilla y protección, pero después la fruta ha salido toda" (Diario de campo, octubre 2020)

Gran parte de la población que ha permitido que "toda la fruta salga" no ha venido de otros sectores, sino que son trabajadores asiduos al sector que, como comentábamos, muchas veces son trabajadores itinerantes, que a través de migraciones circulares están presentes en distintas cosechas. Estas personas, por su grado de dependencia a la estacionalidad de las cosechas y por su propia condición de migrante, no siempre dispone de las necesidades básicas necesarias para cumplir con las exigencias del sector del fruto rojo. El discurso de Lamine, un chico de Mali trabajador del fruto rojo que actualmente se encuentra trabajando en la industria del cítrico, da cuenta de cómo muchos de sus compañeros en esta campaña han estado como él ha estado anteriormente, pero con el agravante de trabajar durante una pandemia sin casa, luz, ni agua, descansando en una chabola.

"Muchos piensan que estamos ahí en chabola por gusto, pero si tú das diez viviendas hoy, hoy se ocupan. Si me das veinte, mañana están ocupadas las veinte. Es que muchos piensan que estamos ahí por gusto y no, así no. Todo el mundo no tiene la misma oportunidad que yo. Yo llevo mucho tiempo aquí, yo ya conozco a mucha gente y eso me ha ayudado a conseguir vivienda, pero hay gente que viene solo tres meses y si hay para compartir, perfectamente, pero si no hay para compartirlas, no pueden alquilar una solo y tampoco hay pisos. Muchos no quieren alquilarlo por nosotros" (Entrevista 3) ${ }^{7}$.

7. Entrevista 3: realizada a Lamine en Lepe. 23 de octubre de 2020, 18:30 p.m. 60:03m. Grabadora de voz. 
El relato de Lamine muestra las complicaciones de establecer un proyecto de vida básico como es el tener acceso a una vivienda de forma prolongada. Esto deriva de la estacionalidad de las temporadas, pero también de su origen racial. La antropóloga Menara Lube (2013) sostiene que el camino que conduce al inmigrante hacia la vivienda precaria y no registrada oficialmente repercute en una segregación que es política, determinando el hacinamiento y la infravivienda que a su vez restringe el acceso a unos derechos sociales básicos.

Lamine muestra una conciencia clara de la esencialidad del trabajo en el campo como sostén de la alimentación de la población y reconoce así el mérito desarrollado por quienes han formado parte del trabajo:

"Mientras la gente estaba en casa, nosotros hemos estado trabajando para que el resto pueda vivir. Si nosotros no trabajamos, los empresarios no ganan; y no solo eso, que si no trabajamos no llevamos alimento a su mesa. El trabajo que hacemos nosotros es trabajo primario, podemos quedarnos en casa, pero tenemos que comer. Hasta ahora no teníamos miedo. Por ejemplo, si se me diagnostica que tengo covid, mi grupo tiene que parar, y si mi grupo para, la finca donde trabajamos ¿qué pasa? La fruta se pudre y si se pudre la fruta, a quien necesita comprarla para comer no llega. Si yo no trabajo, la fruta no llega al mercado, si no llega al mercado, no llega a la mesa. Hay que preocuparse no solo por mí, hay que preocuparse por los demás porque si yo no trabajo, la fruta no llega al mercado" (Entrevista 3).

Pone en valor la esencialidad de los colectivos migrantes y de los colectivos migrantes sin papeles a la vez que señala el estado de desigualdad en el que se encuentran:

"Nosotros es que como no tenemos tantos defensores, sabemos que no hablamos bien o sabemos que somos gente que no estamos en regla $y$, hombre, nosotros deberíamos llamar a la policía por algunas cosas, pero no podemos llamar a la policía porque somos gente que no tenemos documentación. El covid está frenando, pero tenemos que manifestarnos. Ya no nos vamos a cruzar de brazos porque antes muchos no tenían documentación ni sabían hablar, pero ahora muchos tenemos papeles y tenemos que hablar porque no hay dos razas, hay una raza, la raza humana" (Entrevista 3).

Como expone Chadia Arab (2020), no regularizar a los extranjeros fomenta el trabajo ilegal, la explotación y la violencia. Desde esta posición desigual, Lamine expone cómo se ha gestionado en una pandemia una necesidad tan básica como el agua para los trabajadores y trabajadoras que se encuentran habitando infraviviendas en temporada:

"Es que nosotros ahí no entendemos por qué para contratar cisternas para llevar agua puedes poner un punto de agua a cada chabola. Por ese dinero prefieren gastar dinero para nada porque el Estado le ha dado dinero y lo van a gastar por tontería. 
No lo gastan para hacer cosas reales porque van a gastar de una manera que no le sirve ni a nosotros ni a ellos y después del estado de alarma se acabó. La gente sigue buscando agua donde antes en el cementerio y hay un punto de agua ahí en la rotonda" (Entrevista 3)

En la sesión de 15 de abril de 2020, el consejo de Gobierno aprobó unas ayudas extraordinarias para los municipios donde existen asentamientos habitados por personas inmigrantes trabajadoras de las campañas agrícolas con un monto de 2.297.160€ para las provincias de Huelva y Almería (Noticias de la Junta de Andalucía, 2020). Para Huelva se destinaron 1.138.599 euros para el reparto de agua potable, saneamiento de basuras y distribución de alimentos y materiales higiénico-sanitarios. La crítica que hace Lamine a la gestión del presupuesto va dirigida a la acción de reparar puntualmente, sin garantizar un cambio de la estructura que pueda permanecer en el tiempo, como puede ser un punto de agua fijo.

Los relatos de Ana, Alicia, Lamine, así como los de Juan, Alfredo, Enrique o Jaime muestran la disparidad de puntos de vista dentro de un mismo campo de trabajo, en función del lugar que se ocupe: agricultor, trabajadora, trabajador, blanco, migrante, regularizado o no. Ocupar distintos lugares es lo que permite a la industria agroalimentaria global avanzar en los términos que lo hace. Andalucía desde el sector de los frutos rojos opera como "Norte", respecto a los trabajadores y trabajadoras venidas de otras latitudes, pero también se convierte en "Sur", con un modelo propio del Sur Global donde se acapara y especializa la tierra en muy pocos cultivos, de forma sobre-medida, provocando una explotación de recursos humanos, así como de los ecosistemas para mantener el sobreconsumo de los países del norte global. Lamine y el resto de compañeras trabajadoras del sector muestran además cómo las fricciones previas existentes en su labor no han sido reparadas bajo el término de esencialidad. Han sido y son esenciales para el sostén de la vida, sin embargo, vemos cómo la prioridad de mantener la producción no va de la mano con la preocupación de que tal producción se realice en condiciones dignas.

\section{CONCLUSIONES}

El presente artículo es una propuesta de revisión de cómo se considera en la práctica la esencialidad de ciertos trabajos y personas en situaciones emergentes durante el covid-19. Se pretende mostrar la necesidad de dotar de contenido a "lo esencial" para que tenga una incidencia real. En un país europeo, teóricamente moderno y civilizado, conviven, no obstante, personas en infraviviendas. Personas que cada mañana no se han podido quedar en casa por dos motivos: no tener vivienda y ser esenciales para el resto de la población. La realidad que muestra el discurso de Lamine es una consecuencia directa del modelo de producción agroalimentario descrito durante este texto. Podríamos hablar por tanto de un modelo de empleo que crea desempleo a su vez. La extensión 
cada vez mayor del sector de los frutos rojos provoca un desequilibrio en la organización laboral, las condiciones y el tiempo efectivo de cada jornada, hasta el punto de que, como expone Alicia, es impredecible. Unas condiciones muy laxas que van a ser asumidas por trabajadores y trabajadoras que se encuentren en situaciones de mayor precariedad. Esto se va a traducir generalmente en población migrante. Es necesario discutir la idea simplista, expuesta por los algunos agricultores, de que "la gente no quiere trabajar", pues spues, según las vivencias de las trabajadoras, se trata de un modelo productivo que dificulta la organización de la vida en torno al trabajo.

Cuando hablamos de fricciones de lo esencial, hacemos alusión directa a la falta de revisión de las condiciones en las que se está desarrollando un trabajo de primera necesidad durante una pandemia mundial. Una sociedad sana no debería tener en exclusiva como horizonte alcanzar la máxima producción para seguir manteniendo los niveles de exportación. Una sociedad sana instauraría una red de alojamientos y saneamientos urgentes para que nadie deje de quedarse en casa porque no sencillamente no tenga una vivienda digna, mucho menos alguien considerado esencial. Los discursos de las personas participantes ponen en evidencia que las pésimas condiciones previas al covid-19 han seguido estando presentes durante el estado de alarma, tras el estado de alarma y en la actualidad. Es decir, que no ha habido cambios sustanciales en las condiciones socio-laborales. Por el bien tanto de productores como de trabajadoras y trabajadores, como muestran sus relatos, una producción más sostenible ayudaría a valorizar los precios, respetar los ecosistemas y dignificar las jornadas de trabajo.

Ni una pandemia mundial ha podido visibilizar realmente lo esencial, pues cuando se naturalizan incluso en un estado de alarma las fricciones descritas, a lo que atendemos es a un intento de perpetuación de las condiciones anteriores. No siempre lo que se nombra existe; en este caso, lo esencial no está siendo tratado como tal. La antropología tiene la capacidad de adentrarse en un análisis que cuestione estos y otros procesos sociales y su deber es servir a la comprensión crítica de los mismos. 


\section{REFERENCIAS BIBLIOGRÁFICAS}

Albán, Adolfo (2010) "Comida y colonialidad. Tensiones entre el proyecto hegemónico moderno y las memorias del paladar". Calle 14. Revista de Investigación en el Campo del Arte 4(5): 10-23.

Arab, Chadia (2020) Las señoras de la fresa. La invisibilidad de las temporeras marroquíes en España. Madrid: Ediciones del Oriente y el Mediterráneo.

Castillero, Soledad (2019) "Cooperación consciente. Alimentación, educación y construcción. El comedor escolar como eje de inflexión en las agriculturas familiares campesinas". En Amparo Novo, Celia Fernández y Héctor Silveira (coord.) Agricultura familiar y derecho a la alimentación. Reflexiones desde España, América Latina y El Caribe. Oviedo: Ediciones de la Universidad de Oviedo, pp. 113-120.

Contreras, Jesús, y Mabel Gracia (2005) Alimentación y cultura:perspectivas antropológicas. Barcelona: Ariel.

Delgado, Manuel (2013) “Introducción a la economía de Andalucía”. En Moreno, Isidoro y Manuel Delgado (ed.) Andalucía: una cultura y una economía para la vida. Sevilla: Atrapasueños y Autonomía Sur, pp. 75-114.

Entrena, Francisco (2008) "Globalización, identidad social y hábitos alimentarios". Revista de Ciencias Sociales, 1(119): 27-38.

Eur.Lex. Legislación de la Unión Europea (2020) “Principio de precaución”. Disponible en: https://eur-lex.europa.eu/summary/glossary/precautionary_principle.html?locale=es [Consultado el 29 de diciembre de 2020].

Fernández García, María Yolanda (2006) "El concepto jurídico indeterminado de 'servicio esencial' en la Constitución española”. Revista de Administración Pública 170: 325-338.

FRESHUELVA (2020) "Mínimo incremento del 2\% en la superficie plantada de frutos rojos para la campaña 2020". Disponible en: https://freshuelva.es/minimo-incrementodel-2-en-la-superficie-plantada-de-frutos-rojos-para-la-campana-2020/_[Consultado el 1 de octubre de 2020].

Gracia, Mabel (2015) Comemos lo que somos: reflexiones sobre cuerpo, género y salud. Barcelona: Icaria.

Gualda Caballero, Estrella (2003) "Agricultura Andaluza y trabajadores extranjeros. Del jornalero andaluz al temporero inmigrante". CENTRA: Fundación Centro Estudios Andaluces. Disponible en: http://public.centrodeestudiosandaluces.es/pdfs/A200303. pdf" http://public.centrodeestudiosandaluces.es/pdfs/A200303.pdf [Consultado el 03 de octubre de 2020]. 
Junta de Andalucía (2020) "Balanza Comercial Agroalimentaria mes de Abril 2020". Disponible en: https://www.juntadeandalucia.es/export/drupaljda/200626_Balanza_ Comercial_Agroalimentaria_Ene-Abril\%202020.pdf” https://www.juntadeandalucia. es/export/drupaljda/200626_Balanza_Comercial_Agroalimentaria_Ene-Abril\%202020. pdf [Consultado el 03 de octubre de 2020].

Lube, Menara (2013) "Inmigración, vivienda e integración social en España. Dilemas, retos y perspectivas". Ecléctica, Revista de Estudios Culturales, 2: 63-77.

Medina, Xavier (2014) Alimentación y migraciones en Iberoamérica. Barcelona: Editorial UOC.

Mignolo, Walter (1998). The cultures of globalization. Durham: University Press.

Moreno Gómez, Jesús, y María Isabel Amado (1999) "Incidencia de los productos americanos en la gastronomía andaluza”. En Ronald Escobedo, Ana de Zaballa y Óscar Álvarez (eds.) Alimentación y gastronomía. Cinco siglos de intercambios entre Europa y América. Pamplona: Newbook Ediciones, pp. 257-265.

Moreno, Isidoro (2013) "Introducción a la identidad histórica, cultural y política de Andalucía". En Moreno, Isidoro y Manuel Delgado (ed.) Andalucía: una cultura y una economía para la vida. Sevilla: Atrapasueños y Autonomía Sur, pp. 15-69.

Moreno, Isidoro y Manuel Delgado (2013) (ed.) Andalucía: una cultura y una economía para la vida. Sevilla: Atrapasueños y Autonomía Sur.

Naciones Unidas (2020) "El hambre en el mundo sigue aumentando, advierte un nuevo informe de la ONU”. Disponible en: http://www.fao.org/news/story/es/item/1152167/ icode/_[Consultado el 3 de octubre de 2020].

Noticias de la Junta de Andalucía (2020) "Aprobadas ayudas de casi 2,3 millones para asentamientos de inmigrantes en Almería y Huelva". Disponible en: http://www. juntadeandalucia.es/presidencia/portavoz/gobiernoaldia/151446/ConsejodeGobierno/ Presidencia/Covid19/Decretoley [Consultado el 3 de octubre de 2020].

Observatorio de precios y mercados (2019) "Frutos Rojos. Últimos precios". Disponible en:https://www.juntadeandalucia.es/agriculturaypesca/observatorio/servlet/ FrontController?action=Static\&subsector=19\&url=subsector.jsp_[Consultado el 3 de octubre de 2020].

Real Academia española RAE (2020). Disponible en: https://www.rae.es [Consultado el 29 de diciembre de 2020].

Reigada, Alicia (2012) “Más allá del discurso sobrela inmigración ordenada’: contratación en origen y feminización del trabajo en el cultivo de la fresa en Andalucía”. Política y Sociedad, 49(1): 103-122. 
(2011) "Reestructuración agraria, migración laboral y feminización del trabajo en Andalucía (España)”. Agricultura, Sociedad y Desarrollo, 8(1): 19-43.

Stuart, Tristram (2011) Despilfarro. El escándalo Global de la Comida. Madrid: Alianza Editorial. 